Case Report

Muhammad Tariq

FCPS Neurosurgery

Assistant Professor

King Edward Medical University, Lahore, Pakistan

Diggaj Shrestha

MS Neurosurgery

Consultant Neurosurgeon

P.T Birta City Hospital, Jhapa, Nepal

Address for correspondence:

Muhammad Tariq

Assistant Professor, Department of Neurosurgery,

King Edward Medical University,

Mayo Hospital, Lahore Pakistan.

Email-mtariqmehr187@hotmail.com

Date submitted : 28/10/2018

Date accepted : 7/5/2019
Nepal Journal of Neurosciences 16:62-64, 2019

\section{Endoscopic Retrieval Of intraventricular Migrated Whole Ventriculoperitoneal Shunt: A Case Report}

Ventriculoperitoneal shunt is associated with different complications. Mechanical complications like blockage, disconnection and distal migration are relatively common. Distal migration of shunt is well documented. But proximal migration of the shunt into the ventricles is very rare with only a few cases reported in literature. Proximal migration of entire shunt in the ventricle, none of them have been proven. In comparison to frontal burr hole, occipital burr hole could facilitate migration due to relatively straight pathway.

Key words: Burr hole, Endoscopy, Hydrocephalus, VP shunt

the skull(Figure B).CT scan brain showed dilated lateral ventricles and a long-coiled tube inside the lateral ventricles(Figure C,D). CSF analysis showed TLC more than 10000 cells $/ \mathrm{mm}^{3}$ with $90 \%$ neutrophils, very high proteins (1812mg/dl) and a low glucose (10mg/dl). Gram staining and culture were negative.

Patient was started with empirical broad-spectrum antibiotics, intravenous levetiracetam and prepared for endoscopy. Under general anesthesia,a right frontal burr hole was doneat the level of coronal suture about $2.5 \mathrm{~cm}$ from the midline. CSF in the ventricles was extremely turbid and visibility was very poor. Irrigation was done until the ventricular wall and tubing could be identified. The tube was gently manipulated, was notadherent to the ventricular wall. It was held with a grasper and gently pulled out through the working channel (Figure-E).

The coiled tube inside the ventricles was found to be the whole shunt assembly of a Chhabra shunt. Previously placed EVD on the left was removed and a new EVD was placed. Antibiotics were continued postoperatively. The baby became more playful, fever gradually subsided and 
VP shunt
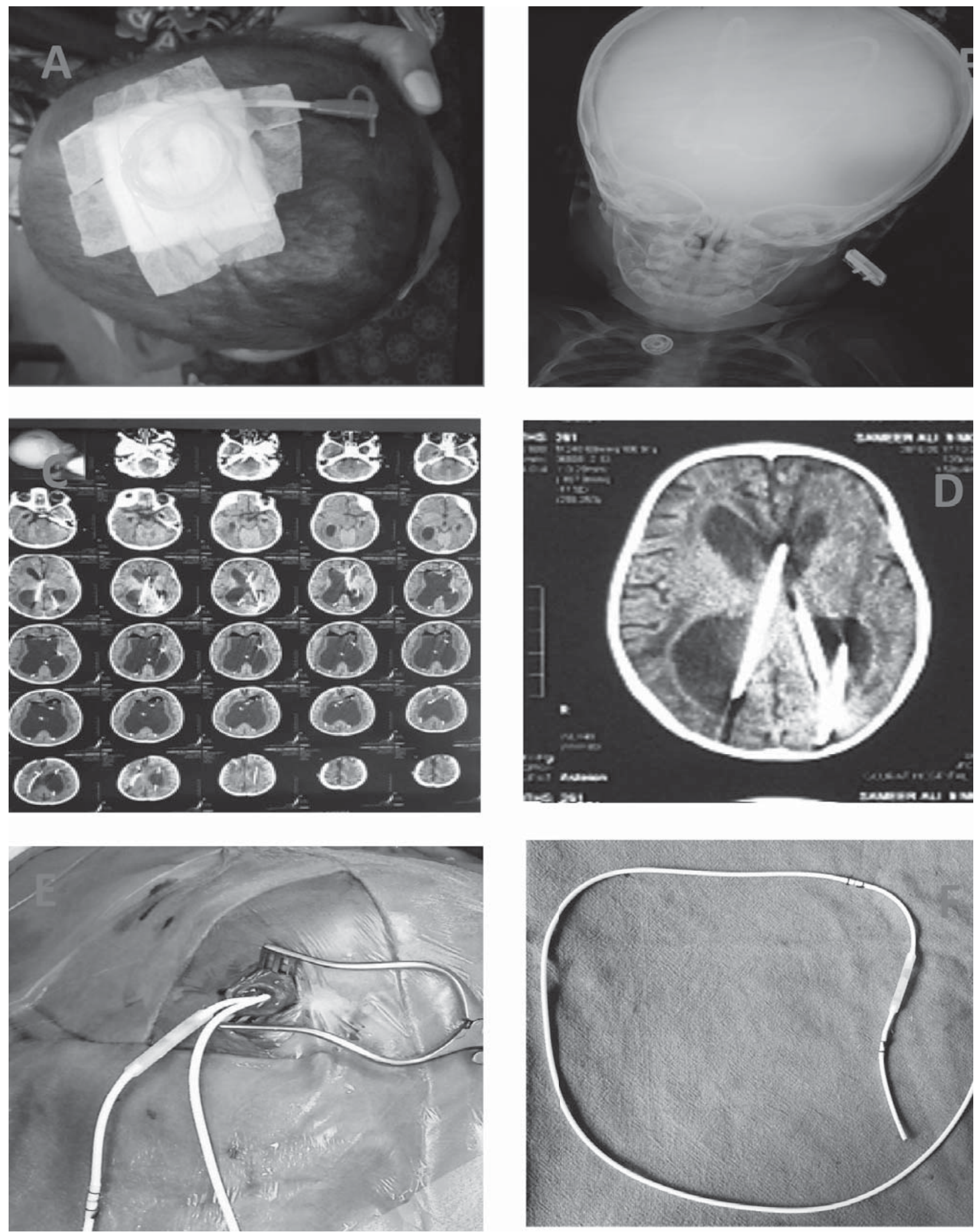

Figure 1. A) Initial presentation of baby with EVD in situ, B)X-ray skull showing shunt in situ, C)Ct scan brain showing dilated ventricles and shunt in situ, D)Ct scan brain plain showing dilated ventricles with shunt in situ with prominent CSF spaces, E)Chhabra shunt being removed F) Whole of the Chhabra shunt removed 


\section{Tariq et al}

CSF cytology gradually returned to normal after 4 weeks. When EVD was closed patient did not develop any signs of raised ICP. Follow up scan showed brain atrophy with dilated ventricular and extra ventricular CSF spaces.EVD was removed and the baby was discharged without any shunt.

\section{Discussion}

Although ventriculoperitoneal shunt placement isthe most commonly used CSF diversion procedure,it is associated with different complications reported in 19 to $47 \%$ of cases. ${ }^{8}$ Obstruction and infection are the commonestamong all the complications. ${ }^{6}$ Other mechanical complications like disconnection of ventricular/peritoneal catheter and migration occur less frequently.

Migration of whole of the shunt system is rare (0.1-4\%) and can happen distally or proximally. ${ }^{3}$ Proximal migration of VP shunt in the lateral ventricle is the rarest complication with very few cases reported in literature. ${ }^{3} \mathrm{~A}$. Malhotra.et all quoted only 20 case reports before their publication. ${ }^{7}$ The shunt used in all the reported cases was either a unisystem shunt or Chhabra shunt.

Although various mechanisms have been suggested for proximal migration of entire shunt in the ventricle, none of them have been proven. In comparison to frontal burr hole, occipital burr hole could facilitatemigration due to relatively straight pathway. ${ }^{4}$ Eljamelet al proposed excessive head movements, positive intraabdominal pressure and negative intraventricular pressure as possible factors for cranial shunt migration. ${ }^{4}$ Garijo et al proposed formation of a cyst around the peritoneal end, with raised pressure inside the cyst causing upward migration. ${ }^{8}$ Mori et al.proposed a large burr hole and improper fixation of the catheter with the periosteum as a probable cause.Pang and Will bergerproposed that straightshunt tubing without bulky valves could facilitate shunt migration.

\section{Conclusion}

This complication can be avoided by using shunts with standard contoured or Salmon Rickham reservoirs. But if Chhabra shunt is to be used due to its cost effectiveness, the authors suggest a frontal burrhole should be used for placement of ventricular catheter and the shunt tubing should be meticulously secured to the periosteum.

\section{References}

1. Acharya R, Bhutani A, Saxena H, Madan V.S . Complete migration of ventriculoperitoneal shunt into the ventricle. Neurological sciences 23:75-77, 2002

2. Ahmed A, Nasser M. Intraventricular migration of VP shunt. Neurosurgical review 18:293-295, 1995

3. Cho K.R , Yeon J.Y , Shin HJ . Upward migration of a peritoneal catheter following ventriculoperitoneal shunt. Journal of Korean Neurosurgical Society 53:383, 2013

4. Eljamel M. S,Sharif S, Pidgeon Ch N .Total intraventricular migration of unisystemventriculoperitoneal shunt. Acta neurochirurgica 136:217218, 1995

5. Huliyappa H, Jaiswal M, Singh S.K. Retrogradepartial migration of ventriculoperitoneal shunt with chamber: Review of causative factors and its prevention. Journal of pediatric neurosciences12:93, 2017

6. Kanojia R, Sinha S.K, Rawat J, Wakhlu A. Unusual ventriculoperitoneal shunt extrusion: experience with 5 cases and review of the literature.Pediatric neurosurgery 44:49-51, 2008

7. Malhotra A, Malhotra M. Ventricular migration of shunt: Chhabra shunt complication.International Surgery Journal 2:706-709, 2016

8. Mubasser A, Muhammad T.Complications of Ventriculoperitoneal shunts and their management: A prospective study.Pakistan Postgraduate Medical Journal. 21 (4), 2010

9. Nadine N, Breedt A. Hydrocephalus. Nursing Care of the Pediatric Neurosurgery Patient. Springer, Cham; 2017

10. Sharma R, Bansal M, Agrawal M, Gupta A .Complete intracranial migration of a ventriculoperitoneal shunt: rare complication of a common procedure. Neurology India 63:106, 2015 